Voix et Images

voixetimages

\title{
Aux marges de la fiction
}

\section{Lucie Robert}

Volume 41, numéro 1 (121), automne 2015

URI : https://id.erudit.org/iderudit/1033968ar

DOI : https://doi.org/10.7202/1033968ar

Aller au sommaire du numéro

Éditeur(s)

Université du Québec à Montréal

ISSN

0318-9201 (imprimé)

1705-933X (numérique)

Découvrir la revue

Citer ce compte rendu

Robert, L. (2015). Compte rendu de [Aux marges de la fiction]. Voix et Images, 41(1), 171-176. https://doi.org/10.7202/1033968ar

Ce document est protégé par la loi sur le droit d'auteur. L’utilisation des services d’Érudit (y compris la reproduction) est assujettie à sa politique d'utilisation que vous pouvez consulter en ligne.

https://apropos.erudit.org/fr/usagers/politique-dutilisation/
Cet article est diffusé et préservé par Érudit.

Érudit est un consortium interuniversitaire sans but lucratif composé de l’Université de Montréal, l’Université Laval et l’Université du Québec à Montréal. Il a pour mission la promotion et la valorisation de la recherche. https://www.erudit.org/fr/ 


\author{
D R A M A T U R G I E \\ Aux marges de la fiction \\ $+++$
}

LUCIE ROBERT

Université du Québec à Montréal

Si la dramaturgie contemporaine se dit postdramatique, voire néodramatique, c'est qu'elle a disposé de l'action mimétique et cathartique telle que la concevait Aristote, mais aussi qu'elle brouille les frontières entre le réel et la fiction, qui est vue comme un monde parallèle imaginaire, extérieur à soi. Les pièces dont il est question dans la présente chronique poussent cette démarche aux frontières de l'écriture dramatique et mettent en œuvre des pratiques inhabituelles au théâtre. Elles reposent sur des jeux de masques où sont superposées les figures de l'auteur, de l'acteur et du personnage, comme dans le cas du theâtre autobiographique, ou des écritures multiples, celle de l'auteur dramatique et celle des témoins ou des archives, ce qui est le propre du théâtre documentaire. Entre les deux, toutes les variations sont possibles. Il y a là comme une quête d'authenticité, voire de vérité, devant un monde fait d'incertitudes. Aux questions soulevées, il faut apporter des réponses qui seront parfois celles du public désormais appelé à intervenir aussi directement que possible. Renoncer à la fiction ne suppose toutefois pas l'entier déni de l'invention et c'est donc dans la composition, le montage et l'écriture que se trouve la signature de l'auteur.

Parmi les productions les plus remarquées sur la scène montréalaise ces dernières années, il faut sans doute souligner d'un trait rouge la trilogie de Mani Soleymanlou ${ }^{1}$, dont la première partie, Un, a été créée le 13 novembre 2012 au Théâtre La Chapelle à Montréal, dans une production d'Orange Noyée. Un a été suivi de Deux, créé le 24 septembre 2013, puis de Trois, le 2 juin 2014. La trilogie, mise en scène et interprétée par l'auteur, a été reprise au Festival d'Avignon à l'été 2014 puis, l'automne suivant, au Théâtre d'Aujourd'hui. Ce qui réunit les trois volets de la trilogie est le «Je, Mani Soleymanlou, le personnage principal de cette histoire [...]» (13). L'intention autobiographique est évidente, inscrite dans le récit qui revient sur l'enfance du personnage à Téhéran puis à Paris et à Toronto, ainsi que sur ses premières années

1 Mani Soleymanlou, Trois [précédé de Un et de Deux], Québec, L'instant même, coll. «L'instant scène», 2014, $166 \mathrm{p}$. 
d'adulte, vécues à Ottawa et enfin à Montréal, où il poursuit ses études à l'École nationale de théâtre du Canada. Se pose alors d'emblée la question identitaire:

\author{
Je résume. \\ L'Iran, on me l'a arraché. \\ En France, j'étais Iranien. \\ À Toronto, j'étais pendant quelque temps un Français-Iranien, ensuite un Canadien \\ who quickly became Canadian. \\ À Ottawa, j'étais un Torontois-Français-Iranien. \\ À Montréal, je suis un Torontois-Arabe-Iranien qui a vécu en France et à Ottawa... \\ et aujourd'hui on me dit: heille mon gars t'es Québécois! (18)
}

Québécois, Soleymanlou l'est-il vraiment, et à quelles conditions? Là est la question lancinante qui traverse le texte de la première partie, qui propose aussi bien une scène intitulée "L'Iran pour les nuls», racontant un pays que l'auteur a peu connu, mais qui reste au fondement de son identité, qu'une série de scènes intitulées «Les lundis découvertes», où il rappelle ses débuts d'acteur. À certaines remarques, on comprend que l'écriture a surgi de la contestation des résultats des élections iraniennes de 2009, dont les images ont été diffusées à la télévision, et dont l'auteur a entendu l'écho au printemps 2012 : «Moi qui, un peu comme toi, suis sorti dans la rue en 2012» (46).

Deux puis Trois montrent l'élargissement du questionnement à l'origine de la trilogie. Deux reprend "le même principe que le début de Un» (53) - l'auteur repart de son enfance à Téhéran -, mais il intègre quelques-uns des commentaires entendus à la création de Un et répond à certaines des questions soulevées alors par le public ou par la critique. Cependant, l'élargissement tient avant tout à l'introduction du second personnage, Manu, double de Mani, rôle écrit et joué par Emmanuel Lalonde-Schwartz, né d'une mère francophone et d'un père juif laïc. S'ensuit un dialogue où se confrontent ces identités composées, aux paramètres parfois antagoniques (en particulier quand surgit la question israélienne). Trois va plus loin en formant un jeu choral de quarante-six personnes qui rejouent les divers épisodes de Un et de Deux, mais en introduisant chaque fois les caractéristiques de leur identité propre, contestant une affirmation ou prolongeant un épisode. À mesure que le texte progresse, les répliques deviennent de courts récits qui s'entrechoquent. À la fin, Mani revient en scène seul et récite un texte en innu, hommage au premier immigrant en terre d'Amérique.

On reste agacé par le tissu de clichés qu'énoncent les répliques ou les scènes prises une à une. Ce n'est là toutefois qu'un effet de surface, car le texte repose sur la présence en scène de personnages essentiellement dialogiques, c'est-à-dire que chacun vit son identité d'abord comme un conflit intérieur, dont il s'agit de démêler les souches avant de composer, avec les autres, une mosaïque identitaire aux aspérités pas toujours émoussées. On reste aussi un peu songeur devant ce texte, dont le caractère personnel, voire intime, rend peu probables les nouvelles productions. Les didascalies sont en effet toutes écrites à la première personne comme s'il s'agissait d'abord de rendre compte d'un spectacle particulier. Pourtant, avec générosité, 
l'auteur annonce très vite: «Si vous désirez reprendre la pièce [...], vous êtes libre de faire ce que vous voulez.» (12) À la fin, conscient de s'adresser d'abord à un lecteur, il écrit: «Merci d'avoir pris le temps de nous lire.» (166)

Soleymanlou n'est évidemment pas le premier à tenter l'autobiographie dramatique, bien que le genre soit peu pratiqué. Et l'on se demande, chaque fois, pourquoi un auteur éprouverait le désir ou le besoin d'étaler ainsi sa vie. Car l'enjeu de l'autobiographie, au thêâtre, ne saurait être le même que celui de l'autobiographie publiée sous la forme d'un livre. La fragmentation en vingt ou trente scènes, nécessairement brèves, rend plus difficile l'introspection et l'on ne saisit pas toujours facilement l'intention ou l'unité du propos. Tel est le problème que soulève la pièce d'Olivier Kemeid, Moi, dans les ruines rouges du siècle², créée le 10 janvier 2013 au Theâtre d'Aujourd'hui dans une mise en scène de l'auteur. La pièce repose sur une idée originale de Sasha Samar, comédien montréalais d'origine ukrainienne, qui interprète son propre personnage et joue sa propre vie: «j'essaie de savoir qui je suis» (18), ditil. Le prologue annonce: "Je suis né le 12 février 1969 en Ukraine soviétique.» (9) Suivent les grands événements de l'enfance. Nous sommes dans l'ordre du récit où Vassili, le père, raconte des souvenirs que Sasha, adulte, essaie de comprendre et de mettre bout à bout. La première partie repose ainsi sur le point de vue du père, blessé dans un accident de mine, qui, depuis le départ de sa femme, vit seul avec son fils, toujours en cavale, par crainte d'en perdre la garde. Le découpage suit quelquesuns des grands événements de l'actualité: la mort de Youri Gagarine, la «série du siècle» au hockey, les Jeux olympiques. Une seconde partie concerne l'adolescence. Sasha est constamment à la recherche de sa mère, mais Vassili multiplie les obstacles. Alors que Sacha étudie à l'école de théâtre, son père s'engage comme liquidateur à Tchernobyl, sous la promesse d'un logement décent et d'une bourse d'études pour son fils. Il survivra encore quelques années, le temps que son fils termine ses études et son service militaire au Kazakstan. À la fin de la pièce, nous sommes en 1994. Sasha a enfin retrouvé sa mère, mais elle meurt peu après. Il se marie et émigre au Canada, où est né son fils, à qui, sans doute, seront livrées ses mémoires. Ce qui frappe surtout, à la lecture, est l'absence de réflexion politique autour des événements pourtant désignés par le titre. Que sont en effet «les ruines rouges du siècle», sinon ce rappel de l'Union soviétique (du point de vue ukrainien, c'est-à-dire depuis les grandes famines vécues par le grand-père jusqu'à l'indépendance du pays, deux mois après la mort de Vassili) et de sa chute? La pérestroïka ne se lit qu'à la découverte de la musique occidentale, Pink Floyd ou Donna Summer, et au détour d'une phrase qui n'appelle pas de réponse: «Dans la poussière des gravats je regarderai le siècle s'écrouler sous mes pieds.» (99) La mort des parents de Sasha apparaît donc comme la clôture d'un siècle aux enjeux personnalisés. Mais on ne saura jamais ce qui a motivé son départ.

2 Olivier Kemeid, Moi, dans les ruines rouges du siècle, idée originale de Sasha Samar et Olivier Kemeid, Montréal, Leméac, coll. «Thêâtre», 2013, 110 p. 
Coller à la vérité du témoignage est peut-être ce qui compte après tout. Et l'on saluera l'originalité de l'approche d'Anne-Marie Olivier, dont le plus récent opus, Faire l'amour ${ }^{3}$ est construit «à partir de témoignages obtenus auprès de gens de tous âges et de tous horizons » (9) au moyen d'un questionnaire reproduit à la page 14. Le texte, créé au Théâtre Périscope à Québec le 15 avril 2014, dans une mise en scène de Véronique Côté, et repris à l'Espace GO le 14 novembre de la même année, rappelle la manière spécifique à l'auteure, connue pour ses contes urbains, une des rares femmes à pratiquer le genre. Il ouvre ainsi sur une exhortation, «Bonsoir, bienvenue», et prévient: "Ce que vous entendrez ce soir, ce sont des histoires vraies, toutes vraies» (15). Nous avons donc là une quinzaine d'histoires. Certaines sont présentées sous la forme d'une scène complète assez développée; d'autres, plus brèves, sont réunies en quatre groupes, sous le titre générique de l'«Autel des amours mortes»; quelquesunes enfin sont découpées en fragments disséminés dans l'ensemble. L'on reste séduit par la variété des cas de figure, mais surtout par la légèreté de ton qu'empruntent les personnages pour faire état d'événements aussi intimes, heureux ou malheureux, de leur vie ou de celle de leurs proches: un couple fait connaissance en s'embrassant, un ado court sur la plage de Saint-Tropez pour voir des seins et vit là les quinze plus belles minutes de sa vie, une vieille tante divorce avant de trouver un nouvel amant, une victime d'inceste aime ses enfants et leur fait l'amour à travers sa cuisine, un jeune homme aux vertèbres brisées se considère chanceux d'avoir trouvé une amoureuse, une jeune lesbienne doit son coming-out à une femme hétérosexuelle mais libre. Les scènes plus soutenues renvoient plutôt au «triangle, comme une forme parfaite» (78) - telle celle de la jeune femme qui traverse l'Argentine avec deux hommes qu'elle tente de rapprocher - ou comme forme imparfaite, quand deux hommes ayant la même maîtresse s'entretuent jusqu'à la noyer, elle. Ainsi, de la naissance du sentiment amoureux jusqu'à sa fin, soit par la rupture, soit par la mort, sont explorées quelques-unes des diverses situations possibles, mais toujours pour mettre en valeur l'intensité du moment et pour célébrer la vie.

L'occasion m'est rarement donnée de faire allusion au théâtre québécois qui s'écrit en anglais, et j'apprécie la traduction que propose Fanny Britt du texte d'Annabelle Soutar, Grains. Monsanto contre Schmeiser ${ }^{4}$, créé en français le 4 septembre 2012 au Thêâtre La Licorne dans une mise en scène de Chris Abraham et joué en tournée canadienne depuis. La pièce appartient à un genre rarement pratiqué, le théâtre documentaire, dont la principale caractéristique est sans doute de mettre en scène un événement de grande importance et de rendre la parole aux divers intervenants. C'est ainsi qu'avaient procédé déjà Jean Provencher et Gilles Lachance, dans Québec,

3 Anne-Marie Olivier, Faire l'amour, Montréal, Atelier 10, coll. «Pièces», 2014, 116 p.

4 Annabelle Soutar, Grains. Monsanto contre Schmeiser, théâtre documentaire, traduit de l'anglais par Fanny Britt, Montréal, Écosociété, 2014, 174 p. 
printemps $1918^{5}$, créée au Trident à Québec en 1973, portant sur la première crise de la conscription, à l'origine de la Loi canadienne sur les mesures de guerre. L'enquête de Soutar, dont il est question ici, rappelle le procès qu'a intenté en 1998 une multinationale de l'agriculture, Monsanto, contre un fermier de la Saskatchewan, à qui elle reprochait d'avoir utilisé ses graines de canola modifiées génétiquement sans payer ses droits alors que le fermier soutenait n'être pas responsable des graines que le vent avait soufflées sur sa terre. On se rappellera peut-être cette histoire, qui est aussi celle «de la première poursuite judiciaire au Canada en matière de protection des graines génétiquement modifiées» (20). Écrite à partir de témoignages recueillis par l'auteure et du compte rendu textuel du procès, la pièce est divisée en deux parties. La première pose les faits, convoque les témoignages du fermier, Percy Schmeiser, de son avocat, d'une militante anti-OGM, d'experts et de contre-experts et se termine sur le verdict de culpabilité rendu par la Cour. La deuxième partie porte sur les retombées de ce premier jugement, qui suscite débats et actions affirmant le caractère négatif des OGM sur l'organisme humain. L'affaire prend alors une dimension internationale qui vaut à Schmeiser le prix Mahatma-Gandhi. Force est à l'auteure d'admettre que l'état embryonnaire de la recherche scientifique en ce domaine laisse tout le monde dans la plus grande confusion. Dans un ultime jugement, la Cour suprême du Canada tranchera d'ailleurs encore en faveur de Monsanto, mais le fermier n'aura pas à payer les frais du procès. La pièce se clôt sur cette inconlusion quant à la cause elle-même, mais - et là est sans doute l'intérêt premier de ce type de thêâtre - elle oblige le public à adopter la posture du jury et à reconstituer, à son propre profit, l'ordre et le sens de l'événement.

Je m'en voudrais de terminer cette chronique sans souligner la parution de l'essai que Jennifer Drouin consacre aux adaptations du thêâtre de Shakespeare en français au Québec, Shakespeare in Québec. Nation, Gender, and Adaptation ${ }^{6}$, essai tiré d'une thèse soutenue à l'Université McGill. Après avoir recensé une trentaine d'adaptations en français, certaines plus connues (celles de Robert Gurik et de Michel Garneau, par exemple), d'autres moins, elle soutient que, depuis 1960, Shakespeare doit être considéré comme une référence encore plus essentielle que Molière sur la scène québécoise. Dans cet ensemble, elle découpe une première époque, où les adaptations ont en commun d'éliminer les personnages féminins, tout en faisant du viol la métaphore des relations entre le Canada et le Québec. Le Lear (1977) de JeanPierre Ronfard inaugurerait une seconde époque, où l'émergence de personnages féminins forts pourrait sauver l'État de la décadence et régénérer la nation. Une troisième époque remet en question ces modèles nationalistes, voire souverainistes. Les adaptations sont alors signées par des femmes, des homosexuels ou des autochtones,

5 Jean Provencher et Gilles Lachance, Québec, printemps 1918, Montréal, L'Aurore, coll. «Entre le parvis et le boxon", 1974, 155 p.

6 Jennifer Drouin, Shakespeare in Québec. Nation, Gender, and Adaptation, Toronto, University of Toronto Press, 2014, 286 p. 
bref, par tous les exclus des adaptations antérieures. L'auteure conclut que les adaptations québécoises de Shakespeare restent radicalement distinctes des adaptations canadiennes contemporaines, témoignant d'une réappropriation volontairement irrévérencieuse et mise au service de la décolonisation de la nation, bien que celle-ci repose sur ce que l'auteure nomme un "nationalism's gender trouble», expression difficile à traduire, qui n'en désigne pas moins l'une des pierres d'achoppement contre lesquelles trébuche, à répétition, la politique québécoise. 